\title{
Identification and Verification of Differential Display cDNAs Using Gene-Specific Primers and Hybridization Arrays
}

BioTechniques 24:1018-1026 (June 1998)

\author{
K.J. Martin, C.-P. Kwan, M.J. \\ O'Hare', A.B. Pardee and R. \\ Sager \\ Dana-Farber Cancer Institute, \\ Boston, MA, USA and \\ ${ }^{1}$ LICR/UCL Medical School, \\ London, England, UK
}

\begin{abstract}
An accurate and streamlined approach to differential display (DD) band identification and verification is described. To minimize false positives, the strategy avoids the use of impure Northern blot probes obtained from PCR-amplified DD bands. To increase throughput, the cloning of $D D$ bands is replaced by a gene-specific primer approach, and hybridization arrays are used in place of Northern blots. In summary, DD bands obtained with long primers were directly sequenced to allow the design and synthesis of gene-specific primers, which were then used to PCR-amplify homogeneous probes for the verification of expression patterns by hybridization array analysis. Differential expression of 60 of the 63 genes tested was confirmed. Thus, false positives are not inherent to $D D$. The results demonstrate the power of DD used with hybridization arrays to rapidly generate information on expression patterns of differentially expressed genes.
\end{abstract}

\section{INTRODUCTION}

Differential display (DD) is a powerful method widely used for the identification of genes differentially expressed between similar cell types or cell growth conditions (14). This method allows the rapid isolation of tags for differentially expressed genes and requires small amounts of total cellular RNA. However, a drawback to the original method is that in many reports, only about half of the genes identified as differentially displayed were actually verified as such by secondary methods. Furthermore, the process of verification by Northern blot analysis can be time-consuming and laborious when many genes are involved.

We have applied DD to study expression genetics in breast cancer (22, 23). Sixty-five candidate tumor-suppressor genes were selected as those expressed at higher levels in normal human breast epithelial cells than in a metastatic breast tumor cell line. Our initial DD comparisons suggested that approximately $3 \%$ of all expressed genes fit this criterion. Because 10000 15000 genes are expressed in any given cell type (1), we estimate that at least 300 genes must be identified to approach saturation of this system. We describe the revised and streamlined approach we have used to identify and verify differential expression of an initial 60 candidate tumor-suppressor genes. The results show that the methods used do not generate a significant number of false positives. Our approach is complementary to others recently described $(13,20)$ and hence ex- pands on the strategies available for discovery and analysis of differentially expressed genes.

\section{MATERIALS AND METHODS}

\section{Cells, RNA and DD}

$76 \mathrm{~N}$ normal human breast epithelial cells (4) and MDA-MB-435 human breast tumor cells (ATCC, Rockville, MD, USA) were cultured in DFCI-1 medium (4). Normal luminal epithelial and myoepithelial cells were separated from primary cultures of mammoplasty specimens (7). Total cellular RNA was isolated by the guanidinium isothiocyanate method (6).

DD (14) was performed using methods and primers described by Zhao et al. (26). Anchor primers were a series of three 21-mers, each with an EcoRI restriction site, a $\mathrm{T}_{11}$ stretch and a single 3' base, either G, A or C. Arbitrary primers were 18- to 21-mers referred to as the LHA- or OPA-series $(17,26)$. Two additional primers with arbitrary sequences, 15-A (CAGTTTCTTGGGACATAGGA) and 15-B (CTCAGCCGTGCTCCACCTAGC), were synthesized and the combination used to generate bands $15 \mathrm{D}$ and $15 \mathrm{~L}$ from cDNA produced by reverse transcribing total cellular RNA with the anchor

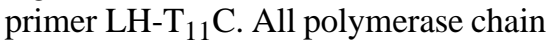
reactions (PCRs) were performed in duplicate and electrophoresed in parallel on extended-format denaturing $6 \%$ polyacrylamide gels using the programmable genomyxLR ${ }^{\mathrm{TM}}$ DNA Sequencer (Genomyx, Foster City, CA, USA) (3). 


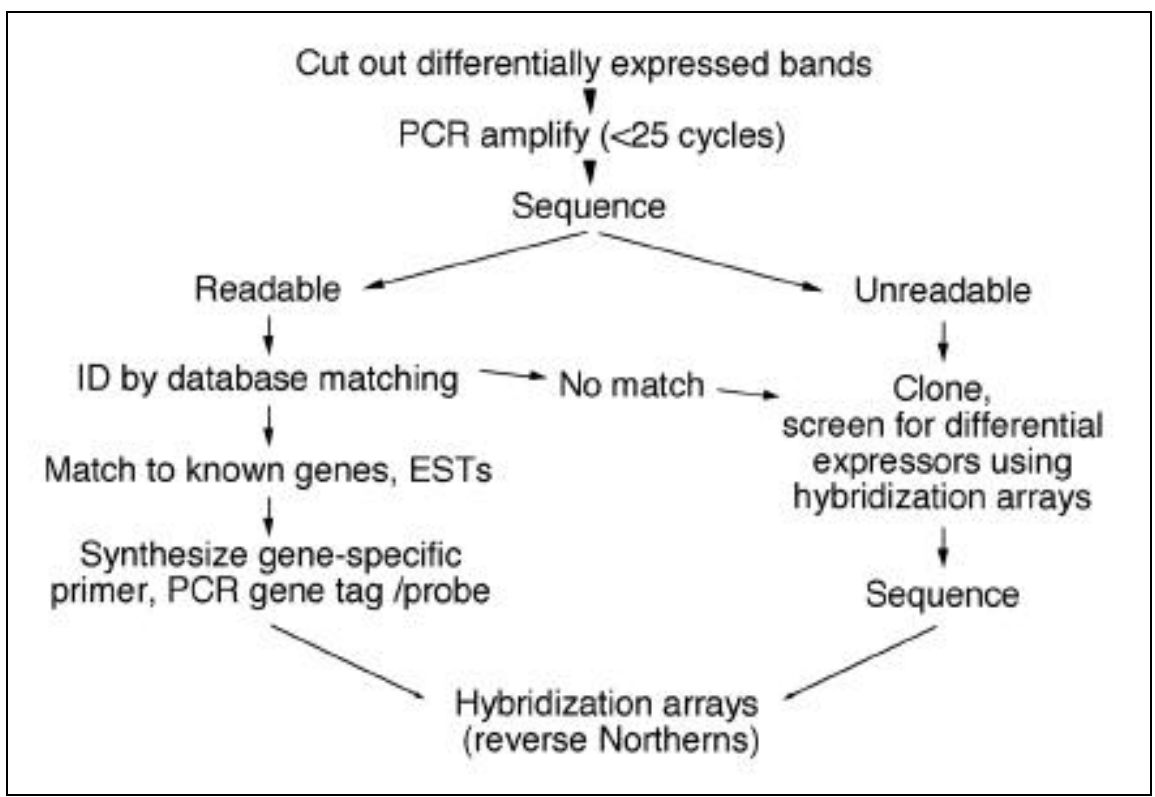

Figure 1. Strategy for identifying and verifying differential expression of DD band cDNAs. Normalspecific bands were excised from DD gels, PCR-amplified and directly sequenced. For those with readable sequences that matched database entries, gene-specific primers were synthesized and used to prepare pure probes for hybridization array analysis. DD bands that produced sequences that were unreadable or could not be matched to database entries were cloned and the clones screened for differential expression by hybridization analysis.

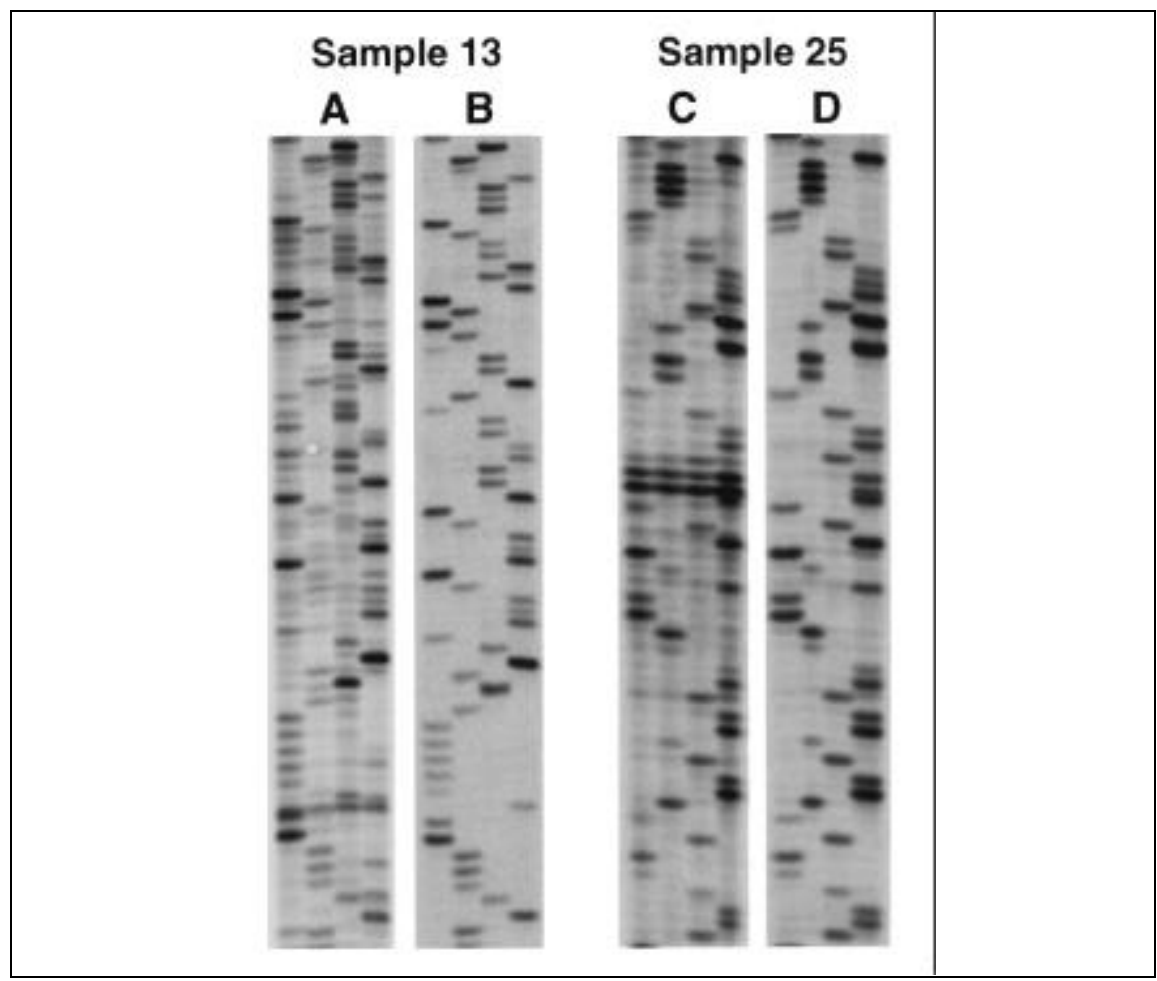

Figure 2. DNA sequencing to access gene tag purity. (A,C) cDNAs derived from DD bands 13 and 25 were PCR-amplified for 25 cycles using the appropriate DD primers, then cycle-sequenced. Sample 13 was sequenced from the anchor primer and sample 25 from the arbitrary primer. (B) Subclone 13e was PCR-amplified for 25 cycles using the DD primers and then cycle-sequenced from the anchor primer. (D) cDNA from band 25 was PCR-amplified for 25 cycles using a gene-specific primer designed to hybridize just inside the DD anchor primer, in combination with the DD arbitrary primer, then cycle-sequenced from the arbitrary primer. Sequencing tracks are shown in the order G, A, T, C. 


\section{DD Band Identification and Verification}

DD bands were identified and their differential expression verified as diagrammed (Figure 1). DNA from excised DD bands was eluted (14), directly sequenced (16) and sequences queried against National Center for Biotechnology Information (NCBI) databases using the Basic Local Alignment Search Tool (BLAST) (2) algorithm. A match was defined as $\geq 96 \%$ identity of bases over a stretch of $\geq 30$ bases. Following database verification, a single gene-specific 20-mer was designed that would hybridize to the DD fragment just inside the arbitrary primer site. This gene-specific primer was synthesized and used in combination with the appropriate DD anchor primer to PCR-amplify a homogeneous probe for Northern blotting (12) or gene tag for hybridization arrays.

Hybridization arrays $(16,19,25)$ were prepared by spotting nylon membranes (ZetaProbe ${ }^{\circledR}$; Bio-Rad, Hercules, CA, USA) in a 96-well format (Bio-Dot ${ }^{\circledR}$ manifold; Bio-Rad) with individual
DNA gene tags. Replicate arrays were then hybridized with ${ }^{32} \mathrm{P}$-labeled cDNA prepared by reverse transcription (RT) of total cellular RNA using a poly $(\mathrm{dT})_{12-18}$ primer. Each gene tag was applied either to four spots as a 3-fold serial dilution series using approximately $0.1 \mu \mathrm{g}$ agarose gel-purified DNA in the first spot (Figure 2) or two spots using approximately $0.1 \mu \mathrm{g}$ DNA in the first spot and $0.003 \mu \mathrm{g}$ in the second. Replicate membranes were hybridized in parallel with different preparations of ${ }^{32} \mathrm{P}-\mathrm{la}$ beled cDNA made from the RNA of specified cell types (16). Membranes were hybridized in bags or roller bottles using standard Northern blot procedures, and signals were detected by phosphor imaging. Signal intensities were calculated for individual spots, backgrounds were subtracted, and values were normalized to the intensity of control gene 36B4, a tag for which was included on each membrane. 36B4 is equally expressed in tumor and normal breast epithelial cells (18). Relative differences were calculated for spots with equal amounts of tag loaded, and differential values were selected from spots with the lowest detectable signals. Equal loading of tags was controlled for by stripping replicate membranes, rehybridizing with labeled cDNA prepared from normal luminal epithelial cells and correcting final values appropriately. All experiments were performed at least twice, and most were performed three times with comparable results. Independent extractions of $76 \mathrm{~N}$ and MDA-MB-435 RNA were used for duplicate tests. A single RNA extraction of sorted cells was used. Geometric means are presented.

DD bands that did not yield readable direct sequences or whose sequences did not match database entries were cloned using standard methods (26) and the subclones screened for differential expression by hybridization dot blotting as described above.

\section{RESULTS}

\section{DD Band Identification and Probe/Gene Tag Preparation}

cDNAs from 104 normal-specific

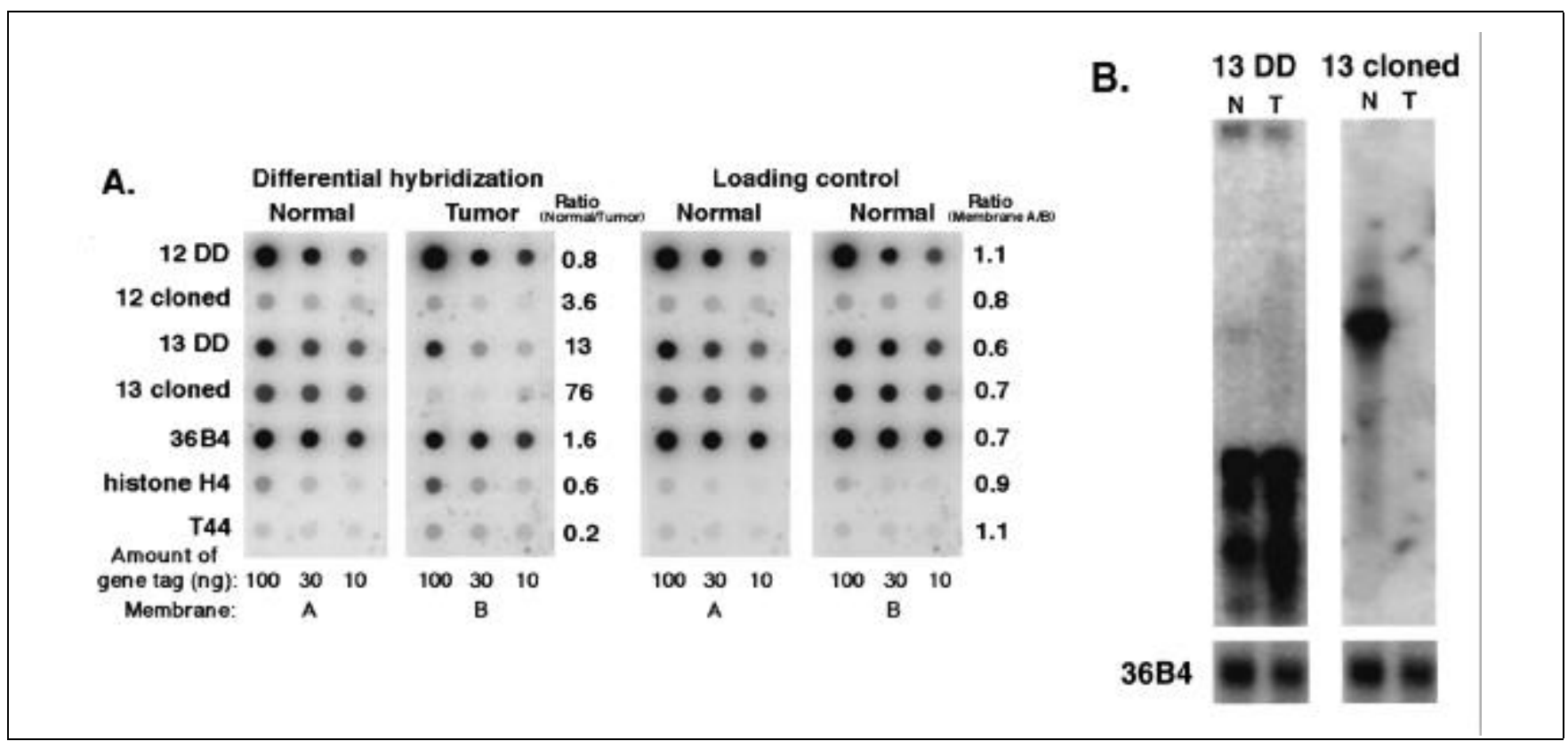

Figure 3. Hybridization array and Northern blot analyses of samples 12 and 13. (A) Hybridization array results. Two membranes, A and B, are replicates loaded with equal amounts of identical gene tags. In the left set (Differential hybridization), the membranes were hybridized in roller bottles with labeled cDNA from cultured 76N breast epithelial cells (Normal) and MDA-MB-435 (Tumor) cells. In the right set (Loading control), both membranes were stripped and then rehybridized with labeled cDNA from cultured $76 \mathrm{~N}$ breast epithelial cells (Normal). Ratios listed to the right of filter sets indicate normal/tumor values. In the case of the differential hybridization, these were normalized by the 36B4 and H4 signals. Tags " 12 DD" and " 13 DD" were derived from DD bands 12 and 13 , respectively, by PCR amplifying for 25 cycles using the appropriate DD primers. Tags " 12 cloned" and " 13 cloned" were generated from cloned bands by PCR amplification as before. 36B4 and H4 represent nondifferentially expressed controls, T44 is a novel up-regulated gene isolated by DD and included as a control. (B) Northern blot results of sample 13. The left panel used a probe made by directly PCR amplifying DD band 13 using the DD primers. The right panel used a probe produced from a clone of band 13. RNA was obtained from cultured 76N breast epithelial cells (N) or the MDA-MB-435 breast tumor cell line (T). 
DD bands were eluted and analyzed following the strategy diagrammed (Figure 1). Fourteen bands (samples 11-22 plus $15 \mathrm{D}$ and $15 \mathrm{~L}$ ) originated from comparisons of $76 \mathrm{~N}$ normal and MDA-MB435 tumor cells. The majority of bands (samples 23-112) were selected from comparisons of sorted breast epithelial cells and the MDA-MB-435 tumor cell line. Sizes of the cDNAs ranged from 150 to $600 \mathrm{bp}$ and averaged $280 \mathrm{bp}$.

Of the 104 bands, $86 \%$ produced readable sequencing tracks, and $92 \%$ of the readable sequences matched nonredundant or expressed sequence tag (EST) database entries. For most of these bands, a nested 20-mer primer was designed that would hybridize near the $5^{\prime}$ end of the DD fragment. This gene-specific primer was used in combination with the appropriate anchor to PCR-amplify a pure gene tag (probe) from the original DD band cDNA. The ability of the gene-specific primer to PCR-amplify a correctly sized DNA fragment confirmed the accuracy of the direct sequence and database match. The DNA fragment was recovered from an agarose gel and used as a probe to verify differential expression by Northern blot or hybridization array analysis. DNA sequencing showed that PCR with a gene-specific primer produced relatively homogeneous gene tags from heterogeneous DD band cDNA (Figure 2). This approach reduced the need to clone and screen DD bands. Seventeen bands that did not produce readable direct sequences or whose sequences did not match database entries were cloned.

The 104 DD bands analyzed represented 67 different genes. One band (band 27) contained multiple differentially expressed genes, although in general, bands were not checked for multiple genes. Fifteen genes were isolated multiple times. Twelve of these were isolated from pairs of adjacent bands that likely represent the paired cDNA strands separated on denaturing DD gels. One gene was displayed as 20 differentially expressed bands on DD tracks generated from a single arbitrary primer (OPA3) used in combination with either of two anchor primers $\left(\mathrm{LHT}_{11} \mathrm{C}\right.$ or $\left.\mathrm{LHT}_{11} \mathrm{G}\right)$. These bands were identified as ribosomal protein S10, though sequences were difficult to read, apparently because of poor PCR amplification of this fragment, which has multiple potential hybridization sites for both the arbitrary and anchor primers.

\section{Complete Analysis of Three DD Bands}

To validate the methods used, three bands were analyzed in detail. Bands 11-13 were directly sequenced, then cloned, and the clones were sequenced. Differential expression was then assayed both by hybridization array analysis and by Northern blotting. Both of these assays were performed using cloned DNA as tags/probes, and the results were compared to those obtained using tags/probes produced by the conventional method of PCR amplifying DD band cDNA using the DD primers. Results were as follows. Direct sequencing identified band 11 as PAI-2, band 12 as a new gene with an EST match and band 13 as laminin $\beta 3$. All eight clones from band 11 matched the PAI-2 sequence, all eight clones from band 12 matched the EST sequence, and band 13 clones included laminin $\beta 3$ cDNAs (13c and 13e) and two additional tags, each encoding a different new gene (13g and $13 \mathrm{~h})$.

Hybridization array analysis performed using gene tags generated from clones $12 \mathrm{a}$ and $13 \mathrm{e}$ confirmed differential expression of these two genes (Figure $3 \mathrm{~A})$. In comparison, when gene tags were prepared by PCR amplification of the DD band using DD primers, samples 12 and 13 each appeared markedly less differential. Control gene tags showed nondifferential expression of 36B4, histone $\mathrm{H} 4$ (Figure 3A) and $\beta$ tubulin (not shown), as well as up-regulation of a novel uncharacterized gene, T44, which was isolated by DD. Membrane stripping and rehybridization with normal ${ }^{32} \mathrm{P}$-labeled cDNA showed that the replicate membranes were equally loaded.

Northern blotting was also performed to compare probe preparation methods. Blots performed using a probe prepared from clone 13e showed differential expression, while blots prepared using probes made by amplifying the DD band with DD primers produced nondifferential results (Figure 3B). For sample 12, Northern blots using a probe prepared from a clone gave 


\section{Cancer Research Techniques}

no detectable signal, while those using probes made by PCR from the DD band with DD primers produced many bands with no obvious differential signals (data not shown). Sample 11 generated no Northern blot signal for either method of probe preparation (data not shown). Thus, hybridization-based assays performed with heterogeneous probes, such as those produced by PCR amplification of DD bands using DD primers, can lead to inaccurate classification of DD bands as false positives.

\section{Results of Hybridization Arrays and Northern Blotting}

Hybridization arrays were used to rapidly screen expression levels of all 65 genes isolated by DD. Representa- tive results are shown (Figure 4A), and the means of repeated assays for all of the samples that produced detectable signals are summarized as bar graphs (Figures 4, B and C). Expression of two genes (bands 97 and 105) was not detected. Differential expression, defined as an expression difference of at least 3 -fold, was confirmed by this analysis for 59 of the 65 genes.

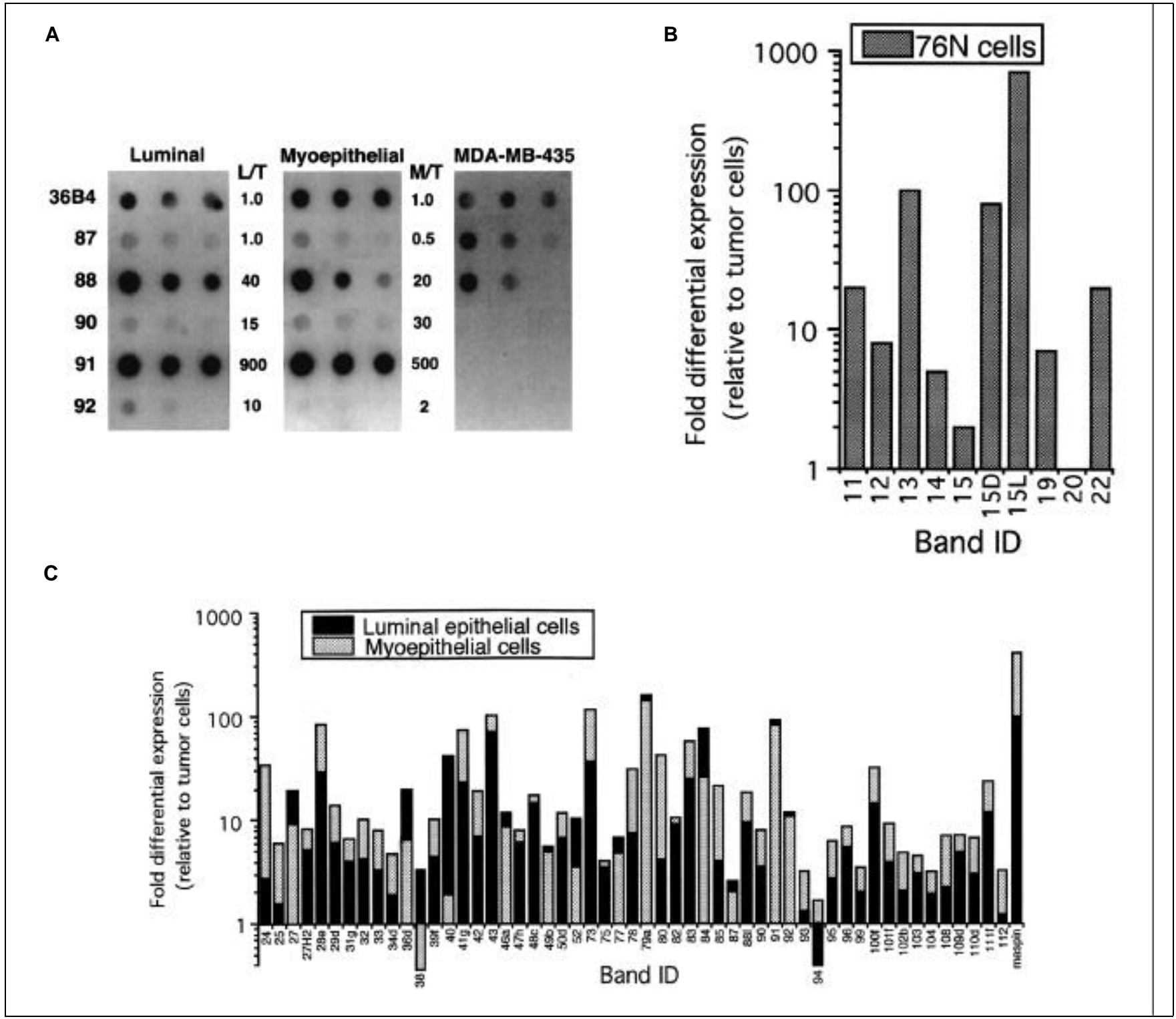

Figure 4. Hybridization array analysis of expression levels of 63 DD-selected genes in normal breast cells relative to breast tumor cells. (A) Representative hybridization array assays. The three membranes shown are replicates loaded with equal amounts of identical gene tags and hybridized with labeled cDNA prepared in parallel from luminal epithelial, myoepithelial and tumor (MDA-MB-435) cells. The image was generated by overnight exposure to film. Band identification numbers are indicated at the left of each row, which is a series of three 3-fold serially diluted gene tags (a fourth spot representing a further 3-fold dilution is not shown). The upper gene tag is the nondifferential control gene 36B4. Sample 87 was a nondifferential false positive, and the others shown were down-regulated in the tumor cells. Luminal/tumor $(\mathrm{L} / \mathrm{T})$ and myoepithelial $(\mathrm{M} / \mathrm{T})$ ratios indicated to the right of the panels were determined by phosphor imaging and normalized to 36B4. (B) Expression of bands 9-22 in 76N relative to MDA-MB-435 cells. (C) Expression of bands 23-112 in myoepithelial and luminal epithelial cells relative to MDA-MB-435 cells. Bars are superimposed. Maspin, a breast tumor-suppressor gene known to be markedly down-regulated in breast tumor cells (23), was included as a positive control. 
The hybridization arrays were performed using conditions that parallel Northern blotting (12) conditions; e.g., hybridization and washing buffers, incubation times and temperatures were identical. The amounts of gene tags used (3-100 ng) encompass the amount of DNA used to generate Northern blot probes $(10-30 \mathrm{ng})$. The validity of the hybridization array results was confirmed by checking 10 genes using Northern blot assays. Northern blotting confirmed differential expression of 9 genes: 13e, 14, 20, 25, 28e, 36d, 46a, and 85 and 100f, while one gene tested, sample 15, was nondifferential (Figure 5). These Northern blotting results were in agreement with the hybridization array results with the exception of band 20 , identified as a false positive by hybridization array analysis but confirmed as a true differential by Northern blotting. Thus, 62 of the 65 genes were verified as differentially expressed using hybridization arrays, while 9 of 10 were verified using Northern blots. These results correspond to false positive rates of 5\% and $10 \%$ for the two assays, respectively.

Sample 28e was unusual in that Northern blots showed two differential bands (Figure 5). A large upper band that ran at the exclusion limit of the gel was specific to normal cells, while a lower band of approximately $5 \mathrm{~kb}$ was specific to the tumor cells. Apparently the larger message, which would have incorporated significantly more $\left[{ }^{32} \mathrm{P}\right]$ dCTP than the smaller, was the predominant species detected by the hybridization array, hence the differential result. This gene may be differentially spliced in tumor vs. normal breast cells so that one of the DD primers did not recognize the shorter transcript. Further analysis of this gene is in progress.

\section{DISCUSSION}

To gain information on breast tumorigenesis, we have applied DD to identify genes expressed in normal breast epithelial cells but not in breast tumor cells. To carry out this study, we developed a new strategy to identify and verify expression patterns of relatively large numbers of genes. The new strategy is composed of previously described techniques assembled in a novel manner. The strategy is streamlined and highly accurate in that it rapidly enabled the identification of 65 candidate breast tumor-suppressor genes and resulted in a negligible rate of false positives.

The DD technique is essentially bipartite, consisting of: (i) RT, PCR and electrophoresis, and (ii) gene identification and verification. Regarding the former, we have used recent improvements reported to reduce false positives to levels of approximately $30 \%$. For example, we used improved DD primers and PCR conditions reported by Zhao et al. (26). All PCRs were performed and electrophoresed in duplicate, and only bands that reproducibly differentially displayed were selected (26). The extended-format programmable genomyxLR electrophoresis apparatus was used to produce reproducible, well-resolved band patterns (3). Our new strategy improves on the latter steps of gene identification and verification by applying previously published techniques in a novel manner. Most importantly, we have avoided the use of impure probes, such as those that result when DD bands are amplified with the original DD primers. This improvement is likely responsible for the reduction in false positives to approximately $5 \%$. Reducing false positives to low levels by improving the verification procedure indicates that in some studies, DD bands might be incorrectly classified as false positives. Hence, high rates of false positives are not inherent to the DD technique.

Two approaches to identifying and verifying DD band cDNAs have been used here. In general, bands that produced legible direct sequence information were pursued with the gene-specific primer approach and were not cloned, while bands that produced poor direct sequences were cloned, and those clones were screened for differential expression. Of the 65 genes isolated, 23 were cloned and 42 were not. We note that considerably more time and effort were required to clone and screen the 23 than to design and synthesize primers for the 42 . Hence, the gene-specific primer approach greatly reduced time and effort by reducing the need to clone and screen.

Direct sequencing is a useful first 
step in the process of DD band identification and verification because it is less sensitive to cDNA heterogeneity than are hybridization assays. Whereas low levels of contaminating cDNAs in probes prepared directly from DD bands can obscure Northern blots if they hybridize efficiently to highly represented RNAs, DNA sequencing is not sensitive to hybridization efficiency or to cellular message representation level. Hence, a $90 \%$ homogeneous DD band will produce a clear direct sequence superimposed on a background signal with an intensity approximately $10 \%$ that of the main signal. Direct sequencing has been previously applied in several DD studies $(15,24)$. Sequence information can readily be confirmed by database matching and used to design a gene-specific primer that hybridizes to the predominant cDNA of a DD band, allowing amplification of a homogeneous probe for use in hybridization assays.

Hybridization arrays also improve the efficiency of DD by streamlining the verification of differential expression. This assay has been used in previous DD studies $(19,25)$ and provides advantages over Northern blotting in terms of time, effort and amount of RNA required per gene assayed, while obtaining at least comparable levels of sensitivity. However, hybridization array assays do not provide transcript size information, which can be used to verify hybridization to the correct mRNA, hence the results are not as informative as those of a Northern blot.

The following conclusions show that the hybridization array assay is sensitive and reliable in its ability to detect changes in mRNA levels in a manner similar to a Northern blot: $(i)$ the assay showed down-regulated expression for $95 \%$ of genes selected as down-regulated using DD when the same two cell types were compared; (ii) results corresponded to those of 9 of 10 Northern blots (the exception, sample 20, was differential by Northern blot and nondifferential by reverse Northern blot assays); (iii) the assay showed appropriate expression of three nondifferential control genes (36B4, histone $\mathrm{H} 4$ and $\beta$ tubulin), an up-regulated control gene (T44) and a down-regulated control gene (maspin); and (iv) loading controls verified that the gene tags were reliably loaded on the replicate membranes.

Hybridization array signals for a given gene showed two types of behavior as the amount of gene tag applied to the membrane decreased. Signals remained constant; i.e., there was no discrimination between the different amounts of gene tag applied to the membrane (Figure 4A, sample 91), or the signal decreased as the amount of gene tag decreased (Figure 4A, sample 88 ). In such cases, lack of discrimination between gene tag amounts did not alter the ability of the assay to detect changes in RNA levels in a manner similar to Northern blotting (Figure 3, $\mathrm{A}$ and B, 13 cloned and 36B4). Hybridization rates might depend entirely on the concentration of $32 \mathrm{P}$-labeled cDNA in solution if cDNA availability is the limiting factor. The differential values obtained by comparing signal intensities for equally loaded spots hybridized with cDNAs from different cell types also showed two general behaviors. Either ratios (normal/tumor) remained constant across the gene tag dilution series (Figure 3A, sample 13, cloned gene tag), or ratios increased as the amount of gene tag bound to the membrane decreased (Figure 3A, sample 13, DD primer method of tag preparation). The latter behavior showed differential expression only at low amounts of gene tag, while higher amounts gave similar signals, apparently because of increased hybridization to contaminants in the gene tag preparation and/or heterologous (nonspecific) hybridization. This demonstrates the importance of preparing membranes with a range of gene tag amounts and obtaining differential values from the spots with the lowest detectable signals.

The types of previously known genes isolated in this study further validate the methods used. Of the 65 different gene tags isolated, 30 matched published sequences of known genes encoding four types of proteins: $(i)$ proteins involved in cell adhesion and communication, including three hormones or cytokines, three protease inhibitors, two extracellular matrix proteins

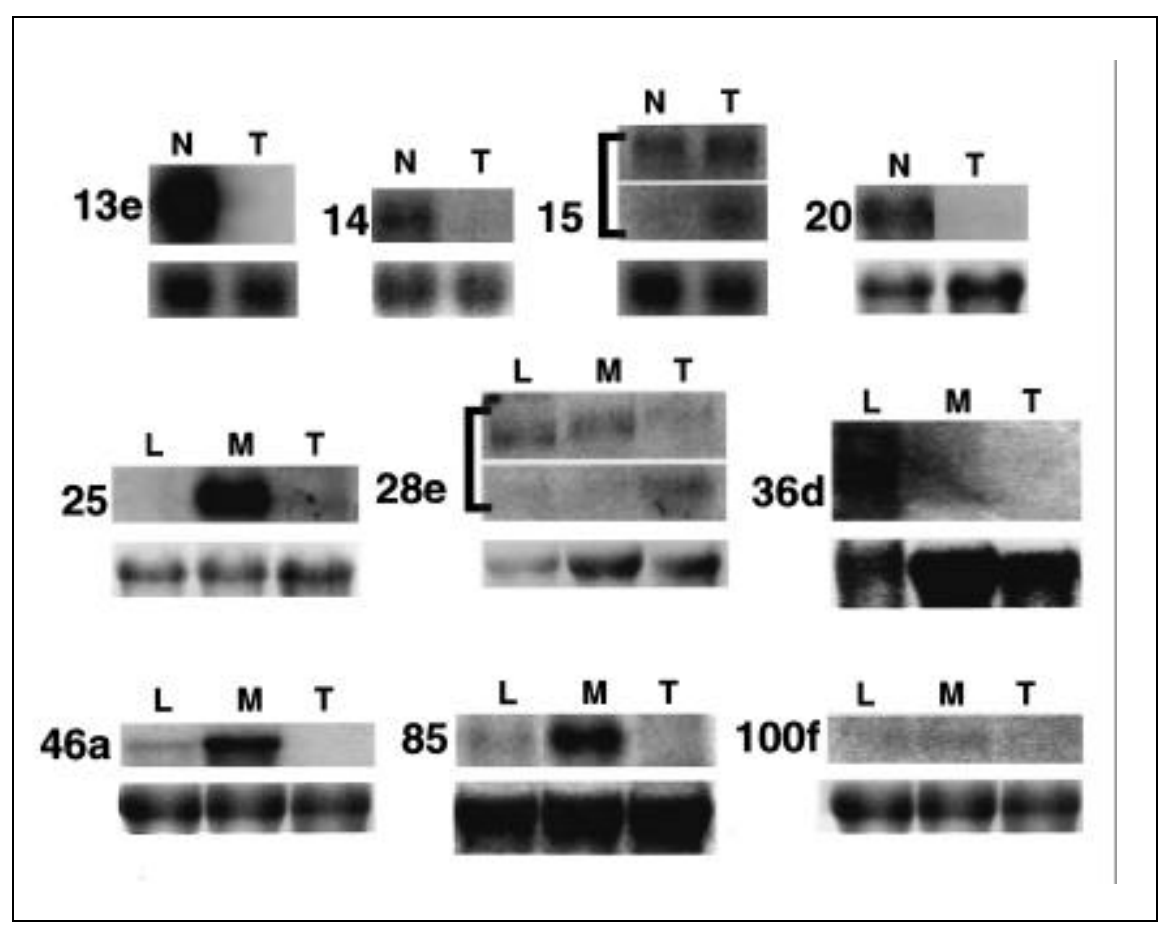

Figure 5. Northern blots of 10 genes selected as down-regulated in breast tumor cells by DD. Upper panels represent gene-specific hybridization, and lower panels are loading controls. For 15 and 28e, two gene-specific bands were observed. The loading control for $13 \mathrm{e}$ and 15 is the $36 \mathrm{~B} 4$ gene and for the remainder is the photo-inverted ethidium bromide signal of 28S rRNA. RNA was obtained from cultured $76 \mathrm{~N}$ breast epithelial cells $(\mathrm{N})$, the MDA-MB-435 breast tumor cell line (T) or immunomagnetically sorted populations of normal breast luminal epithelial (L) or myoepithelial (M) cells. 
and two cell membrane-associated receptor/signal-transduction proteins, (ii) filamentous proteins, including six cytokeratins, (iii) enzymes and (iv) regulatory proteins, including two DNAbinding proteins and a cell cycle inhibitor. These classes of genes identified fit well with our understanding of tumor cells as defective in their ability to respond to growth-regulatory signals from neighboring cells and the extracellular matrix. A number of the individual genes have been previously reported as tumor suppressors and/or as down-regulated in breast and other tumors, e.g., PAI-2 (band 11) (9), 5' nucleotidase (band 43) $(8,21)$ and ribosomal protein S10 (bands 52-72) (10). The activity of cystatin A (stefin A) (band 49), an inhibitor of cysteine protease inhibitors, is reduced in tumors derived from several tissue types and has been proposed to be a tumor suppressor (5). Interferon $\gamma$ (band 90) is a known inhibitor of breast epithelial cell proliferation (11) and is currently used to treat a variety of tumor types. Known genes not previously associated with breast tumor formation were also identified. Further tests will be required to establish the generality with which these genes are down-regulated in tumors.

Results of this study demonstrate the power of DD used in combination with hybridization arrays to obtain relatively large amounts of information on patterns of expression of panels of specifically selected genes in the absence of sophisticated robotics and mechanization. Within the cancer field, this approach is applicable to the development of multifaceted marker systems for screening and categorizing patient tumors in terms of both prognostic and diagnostic information and can be used to gain information on coordinate gene expression and molecular pathways involved in tumorigenesis.

\section{ACKNOWLEDGMENTS}

The authors thank Xiaohong Zhang, Brian Koh and Brian Kritzman for technical assistance. Dr. Sager, recently deceased, was the driving force for the work reported here. Without her enthusiasm and insight, this project would not have been possible.

\section{REFERENCES}

1.Alberts, B., D. Bray, J. Lewis, M. Raff, K. Roberts and J.D. Watson. 1989. Molecular Biology of the Cell. Garland, New York.

2.Altschul, S.F., W. Gish, W. Miller, E.W. Myers and D.J. Lipman. 1990. Basic local alignment search tool. J. Mol. Biol. 215:403-410.

3.Averboukh, L., S.A. Douglas, S. Zhao, K. Lowe, J. Maher and A.B. Pardee. 1996. Better gel resolution and longer cDNAs increase the precision of differential display. BioTechniques 20:918-921.

4.Band, V. and R. Sager. 1989. Distinctive traits of normal and tumor-derived human mammary epithelial cells expressed in a medium that supports long-term growth of both cell types. Proc. Natl. Acad. Sci. USA 86:1249-1253.

5.Calkins, C.C. and B.F. Sloane. 1995. Mammalian cysteine protease inhibitors: biochemical properties and possible roles in tumor progression. Biol. Chem. Hoppe Seyler 376:71-80.

6.Chirqwin, J.M., A.E. Przybyla, R.J. MacDonald and W.J. Rutter. 1979. Isolation of biologically active ribonucleic acid from sources enriched in ribonuclease. Biochemistry 18:5294-5299.

7.Clarke, C., J. Titley, S. Davies and M.J. O'Hare. 1994. An immunomagnetic separation method using superparamagnetic (MACS) beads for large-scale purification of human mammary luminal and myoepithelial cells. Epithelial Cell Biol. 3:38-46.

8.Dao, T.L.K., C. Ip and J. Patel. 1980. Serum sialyltransferase and $5^{\prime}$-nucleotidase as reliable biomarkers in women with breast cancer. J. Natl. Cancer Inst. 65:529-534.

9.Foekens, J.A., F. Buessecker, H.A. Peters, U. Krainick, W.L. van-Putten, M.P. Look, J.G. Klign and M.D. Kramer. 1995. Plasminogen activator inhibitor-2: prognostic relevance in 1012 patients with primary breast cancer. Cancer Res. 55:1423-1427.

10.Frigerio, J.M., J.C. Dagorn and J.L. Iovanna. 1995. Cloning, sequencing and expression of the L5, L21, L27a, L28, S5, S9, S10 and S29 human ribosomal protein mRNAs. Biochim. Biophys. Acta 1262:64-68.

11.Harvat, B.L. and A.M. Jetten. 1996. Gamma-interferon induces an irreversible growth arrest in mid-G1 in mammary epithelial cells which correlates with a block in hyperphosphorylation of retinoblastoma. Cell Growth Differ. 7:289-300.

12.Hirschi, K.K., C. Xu, T. Tsukamoto and R. Sager. 1996. Gap junction genes Cx26 and Cx43 individually suppress the cancer phenotype of human mammary carcinoma cells and restore differentiation potential. Cell Growth Differ. 7:861-870.

13.Kester, H.A., B.M. van der Leede, P.T. van der Saag and B. van der Berg. 1997. Novel progesterone target genes identified by an improved differential display technique suggest that progestin-induced growth inhibition of breast cancer cells coincides with enhancement of differentiation. J. Biol. Chem. 272:16637-16643.

14.Liang, P. and A.B. Pardee. 1992. Differential display of eukaryotic messenger RNA by means of the polymerase chain reaction. Sci- ence 257:967-971.

15.Linskens, M.H.K., J. Feng, W.H. Andrews, B.E. Enlow, S.M. Saati, L.A. Tonkin, W.D. Funk and B. Villeponteau. 1995. Cataloging altered gene expression in young and senescent cells using enhanced differential display. Nucleic Acids Res. 23:3244-3251.

16.Martin, K., C.-P. Kwan and R. Sager. 1997. A direct-sequencing-based strategy for identifying and cloning cDNAs from differential display gels, p. 77-85. In A.B. Pardee and P. Liang (Eds.), Methods in Molecular Biology. Differential Display: Methods and Applications. Humana Press, Totowa.

17.Martin, K.J. and A.B. Pardee.The principles of differential display. In S. Weissman (Ed.), Methods in Enzymology, cDNA Preparation and Analysis. Academic Press, San Diego (In press).

18.Masiakowski, P., R. Breathnach, J. Bloch, K. Gannon, A. Krust and P. Chambon. 1982. Cloning of cDNA sequences of hormone-regulated genes from the MCF-7 human breast cancer cell line. Nucleic Acids Res. 10:7895-7903.

19.Mou, L., H. Miller, J. Li, E. Wang and L. Chalifour. 1994. Improvements to the differential display method for gene analysis. Biochem. Biophys. Res. Commun. 199:564-569.

20.Poirier, G.M., J. Pyati, J.S. Wan and M.G. Erlander. 1997. Screening differentially expressed cDNA clones obtained by differential display using amplified RNA. Nucleic Acids Res. 25:913-914.

21.Rackley, R.R., T.J. Lewis, E.M. Preston, C.M. Delmoro, E.L. Bradley, M.I. Resnick, T.P. Pretlow and T.G. Pretlow. 1989. 5'-Nucleotidase activity in prostatic carcinoma and benign prostatic hyperplasia. Cancer Res. 49:3702-3707.

22.Sager, R. 1997. Expression genetics in cancer: shifting the focus from DNA to RNA. Proc. Natl. Acad. Sci. USA 94:952-955.

23.Sager, R., S. Sheng, A. Anisowicz, G. Sotiropoulou, Z. Zou, G. Stenman, K. Swisshelm, Z. Chen et. al. 1994. RNA genetics of breast cancer: maspin as paradigm. Cold Spring Harb. Symp. Quant. Biol. 59:537-546.

24.Wang, X. and G.Z. Feuerstein. 1995. Direct sequencing of DNA isolated from mRNA differential display. BioTechniques 18:448-453.

25.Zhang, H., R. Zhang and P. Liang. 1996. Differential screening of gene expression difference enriched by differential display. $\mathrm{Nu}-$ cleic Acids Res. 24:2454-2455.

26.Zhao, S., S.L. Ooi and A.B. Pardee. 1995. New primer strategy improves precision of differential display. BioTechniques 18:842850 .

Received 9 July 1997; accepted 16 February 1998.

Address correspondence to:

Dr. Katherine J. Martin

Division of Cancer Genetics

Dana-Farber Cancer Institute and Harvard

Medical School

44 Binney Street

Boston, MA 02115, USA 